ESAIM: M2AN 46 (2012) 187-206

DOI: $10.1051 / \mathrm{m} 2 \mathrm{an} / 2011044$
ESAIM: Mathematical Modelling and Numerical Analysis

www.esaim-m2an.org

\title{
ACCURATE NUMERICAL DISCRETIZATIONS OF NON-CONSERVATIVE HYPERBOLIC SYSTEMS
}

\author{
UlRIK SKRE FJORDhOLM $^{1}$ AND Siddhartha Mishra ${ }^{1}$
}

\begin{abstract}
We present an alternative framework for designing efficient numerical schemes for nonconservative hyperbolic systems. This approach is based on the design of entropy conservative discretizations and suitable numerical diffusion operators that mimic the effect of underlying viscous mechanisms. This approach is illustrated by considering two model non-conservative systems: Lagrangian gas dynamics in non-conservative form and a form of isothermal Euler equations. Numerical experiments demonstrating the robustness of this approach are presented.
\end{abstract}

Mathematics Subject Classification. 65M06, 35L65.

Received August 7, 2010. Revised April 26, 2011.

Published online October 3, 2011.

\section{INTRODUCTION}

\subsection{Systems in conservative form}

Many interesting models in physics and engineering are formulated as systems of conservation laws. Examples include the shallow water systems of hydrology, the Euler equations of gas dynamics and the equations of magneto-hydrodynamics. In one space dimension, these equations can be written as non-linear PDEs of the form

$$
\mathbf{u}_{t}+\mathbf{f}(\mathbf{u})_{x}=0
$$

Here, $\mathbf{u}: \mathbb{R} \times \mathbb{R}_{+} \rightarrow \mathbb{R}^{m}$ and $\mathbf{f}: \mathbb{R}^{m} \rightarrow \mathbb{R}^{m}$ are the vector of unknowns and the flux vector, respectively. The system is completed with suitable initial and boundary conditions. The system is termed hyperbolic if the eigenvalues of the flux Jacobian $\nabla_{\mathbf{u}} \mathbf{f}$ are real. It is well known that solutions of (1.1) develop discontinuities (shock waves) in finite time even if the initial data is smooth. Hence, solutions of (1.1) are sought in the sense of distributions [10]. This definition makes sense for $\mathbf{u} \in L_{\mathrm{loc}}^{1}$ as the flux is in the conservative form and the derivative on the flux can be transferred to the test function.

Weak solutions of (1.1) need not be unique and must be supplemented with additional admissibility criteria or entropy conditions in order to select a physically meaningful solution. The entropy condition is based on the existence of a convex entropy function $S: \mathbb{R}^{m} \rightarrow \mathbb{R}$ and entropy flux function $Q: \mathbb{R}^{m} \rightarrow \mathbb{R}$ such that

$$
\nabla_{\mathbf{u}} Q(\mathbf{u})=\left\langle\mathbf{v}, \nabla_{\mathbf{u}} \mathbf{f}(\mathbf{u})\right\rangle
$$

\footnotetext{
Keywords and phrases. Non-conservative products, numerical schemes.

1 Seminar for Applied Mathematics, ETH Zürich, Rämistrasse 101, 8092 Zürich, Switzerland. ulrikf@sam.math.ethz.ch; smishra@sam.math.ethz.ch
} 
The entropy variables $\mathbf{v}$ are defined as $\mathbf{v}=\nabla_{\mathbf{u}} S(\mathbf{u})$. Then, the admissible solutions of (1.1) have to satisfy the entropy inequality

$$
S(\mathbf{u})_{t}+Q(\mathbf{u})_{x} \leq 0 .
$$

This inequality holds in the sense of distributions and leads to a stability estimate on the entropy solutions of (1.1). The entropy inequality encodes information about small scale effects like diffusion and dispersion.

\subsection{Systems in non-conservative form}

There are many interesting physical models that cannot be expressed in the conservative form (1.1). Instead, they are written in non-conservative quasilinear form:

$$
\mathbf{w}_{t}+\mathbf{A}(\mathbf{w}) \mathbf{w}_{x}=0 .
$$

Here $\mathbf{A}: \mathbb{R}^{m} \rightarrow \mathbb{R}^{m \times m}$ is a matrix-valued function. Note that the conservative form (1.1) can be written in the above form by setting $\mathbf{A}=\nabla_{\mathbf{u}} \mathbf{f}$ as the Jacobian matrix. However, it may not be possible to rewrite (1.4) in the conservative form. The non-conservative system (1.4) is termed hyperbolic if the eigenvalues of $\mathbf{A}$ are real.

Examples of non-conservative hyperbolic systems include shallow water equations with bottom topography [3], gas flows in a duct [15], multi-layer shallow water equations $[1,2,4]$ and multi-phase flows [26].

Analogous to the conservative system (1.1), solutions of the non-conservative system develop discontinuities, even for smooth initial data. Hence, the solutions of (1.4) need to be interpreted in a suitable weak sense. However, a distributional solution of (1.4) is not well-defined as it involves a non-conservative product of distributions.

In [7], Dal Maso et al. defined the non-conservative product $\mathbf{A}(\mathbf{w}) \mathbf{w}_{x}$ in terms of a path connecting left and right states $\mathbf{w}_{L}$ and $\mathbf{w}_{R}$ across discontinuities of $\mathbf{w}$. A path is a $C^{1}$ function $\Phi:[0,1] \times \mathbb{R}^{m} \times \mathbb{R}^{m} \rightarrow \mathbb{R}^{m}$ such that $\Phi\left(0, \mathbf{w}_{L}, \mathbf{w}_{R}\right)=\mathbf{w}_{L}$ and $\Phi\left(1, \mathbf{w}_{L}, \mathbf{w}_{R}\right)=\mathbf{w}_{R}$ for all $\mathbf{w}_{L}, \mathbf{w}_{R} \in \mathbb{R}^{m}$. Without providing details about the definition and properties of the non-conservative product, we would like to point out that the path $\Phi$ is used to obtain a generalized Rankine-Hugoniot relation at a jump discontinuity connecting $\mathbf{w}_{L}$ and $\mathbf{w}_{R}$ with speed $\sigma$ :

$$
\sigma\left(\mathbf{w}_{R}-\mathbf{w}_{L}\right)=\int_{0}^{1} \mathbf{A}\left(\Phi\left(s, \mathbf{w}_{L}, \mathbf{w}_{R}\right)\right) \frac{\partial \Phi}{\partial s}\left(s, \mathbf{w}_{L}, \mathbf{w}_{R}\right) \mathrm{d} s .
$$

Hence, the shock speed and jump relations at a discontinuity depend on the choice of the path $\Phi$.

If the matrix $\mathbf{A}$ can be written as Jacobian, i.e., $\mathbf{A}=\nabla_{\mathbf{u}} \mathbf{f}$, then the non-conservative system (1.4) can be written as a conservative system (1.1) and the generalized Rankine-Hugoniot condition (1.5) reduces to the standard Rankine-Hugoniot condition for (1.1):

$$
\sigma\left(\mathbf{u}_{R}-\mathbf{u}_{L}\right)=\mathbf{f}\left(\mathbf{u}_{R}\right)-\mathbf{f}\left(\mathbf{u}_{L}\right) .
$$

Note that this relation is independent of the choice of path. For stability properties of the non-conservative product we refer to [7].

The weak solutions of (1.4) (based on the non-conservative product of [7]) need not be unique. We assume the existence of an entropy function $S$ and entropy flux function $Q$ such that the solution w of (1.4) satisfies the entropy inequality

$$
S(\mathbf{w})_{t}+Q(\mathbf{w})_{x} \leq 0
$$

(cf. (1.3)). Note that the entropy inequality is still in a conservative form; hence it holds in the sense of distributions. Furthermore, the entropy inequality is independent of the choice of paths. An example of a nonconservative system with an entropy inequality was presented in the context of shallow water equations with bottom topography in a recent paper [9]. The general notion of mathematical entropy for non-conservative systems was discussed in [13] The existence of an entropy solution for non-conservative systems with smallness assumptions on initial data was shown in [7]. 


\subsection{Numerical schemes}

Numerical schemes for the conservation law (1.1) have undergone rapid development in the last few decades. Finite volume (conservative finite difference) methods (see [17]) are frequently employed for approximating (1.1). For simplicity, we consider a uniform Cartesian mesh $\left\{x_{j}\right\}_{j \in \mathbb{Z}}$ with mesh size $x_{j+1}-x_{j} \equiv \Delta x$. The midpoint values are $x_{j+1 / 2}:=\frac{x_{j}+x_{j+1}}{2}$ and the domain is partitioned into intervals $I_{j}=\left[x_{j-1 / 2}, x_{j+1 / 2}\right]$. The time levels are denoted by $t^{n}$ and the time step by $\Delta t$. A standard semi-discrete finite volume scheme can be written as

$$
\frac{\mathrm{d}}{\mathrm{d} t} \mathbf{u}_{j}=-\frac{1}{\Delta x}\left(\mathbf{F}_{j+1 / 2}-\mathbf{F}_{j-1 / 2}\right)
$$

Here, $\mathbf{u}_{j}$ is an approximation to the cell average of the solution in $I_{j}$ and $\mathbf{F}_{j+1 / 2}=\mathbf{F}\left(\mathbf{u}_{j}, \mathbf{u}_{j+1}\right)$ is a numerical flux function, consistent with the flux $\mathbf{f}$ in (1.1). Higher-order schemes based on (1.7) can be designed by standard procedures [17]. The time dependence of (1.7) is suppressed for notational convenience. For this and all subsequent schemes we will use second-order accurate explicit SSP Runge-Kutta time-stepping [11] to obtain a fully discrete scheme.

The design of efficient schemes for the non-conservative system (1.4) is still immature. In [14] Hou and LeFloch showed that for scalar conservation laws, non-conservative finite volume schemes will in general converge to the wrong solution. Similar results were established in [5] for the more general case of non-conservative schemes for non-conservative systems.

An interesting approach to solving nonconservative systems numerically was presented by Karni in [12] in the special case where (1.4) can be rewritten in conservative form (1.1). There, the modified equation of schemes for both systems is computed to second order, and the difference in second-order terms is added as a correction term to the nonconservative scheme. While good results are obtained in numerical tests, the approach has at least two limitations: first, it is dependent on having a conservative form of the system; and second, the computation of the modified equation might be difficult.

In a recent paper [21], Pares has proposed a theoretical framework for designing schemes approximating (1.4). The finite volume (difference) scheme of [21] is of the form

$$
\frac{\mathrm{d}}{\mathrm{d} t} \mathbf{w}_{j}=-\frac{1}{\Delta x}\left(\mathbf{D}_{j+1 / 2}^{-}+\mathbf{D}_{j-1 / 2}^{+}\right) .
$$

The fluctuations $\mathbf{D}_{j+1 / 2}^{ \pm}=\mathbf{D}^{ \pm}\left(\mathbf{w}_{j}, \mathbf{w}_{j+1}\right)$ are assumed to be formally path-consistent, that is, they satisfy

$$
\mathbf{D}_{j+1 / 2}^{+}+\mathbf{D}_{j+1 / 2}^{-}=\int_{0}^{1} \mathbf{A}\left(\Phi\left(s, \mathbf{w}_{j}, \mathbf{w}_{j+1}\right)\right) \frac{\partial \Phi}{\partial s}\left(s, \mathbf{w}_{j}, \mathbf{w}_{j+1}\right) \mathrm{d} s,
$$

with $\Phi$ being the path used in defining the non-conservative product. Fluctuations of the Godunov, Roe and other approximate Riemann solver types have been proposed in [21] and in [19,20].

Recent papers like [2] and [5] have investigated path-consistent schemes in detail and found examples where these do not converge to the solution corresponding to the chosen path. In [2], Abgrall and Karni ask the following questions: (i) how does one go about choosing a path for a given non-conservative system; (ii) once a path is chosen and a path-consistent scheme is defined, does the scheme converge to the solution defined by the path; and (iii) in problems where the correct solution is known without ambiguity, how can one design schemes that converge to the corresponding solution. They explore these pertinent questions for a specific system modeling gas dynamics in Lagrangian coordinates and conclude that path-consistent schemes might not converge to the correct solution. 


\subsection{Scope of this paper}

In this paper, we aim to address the above issues. Our strategy is based on the following ingredients:

(1) Entropy conservative schemes: We design finite difference discretizations of (1.4) such that the scheme satisfies a discrete version of the entropy identity

$$
S(\mathbf{w})_{t}+Q(\mathbf{w})_{x}=0
$$

This is based on the entropy conservative schemes for (1.1) developed by Tadmor in [22,23] and extensively investigated in recent papers $[8,9]$. Entropy conservative schemes for the shallow water equations with bottom topography were designed in [9].

(2a) Physical viscosity: entropy is conserved for smooth solutions of (1.4) but needs to be dissipated at shocks. One method for introducing entropy dissipation is by directly discretizing the underlying convection-diffusion equation for (1.4) by adding the previously ignored physical diffusion operator. An entropy stable discretization of the physical diffusion operator is added to the entropy conservative scheme for (1.4). This strategy has been investigated in the context of the conservative system $(1.1)$ in $[8,24,25]$. We extend it to the case of non-conservative systems in this paper.

(2b) Numerical diffusion: a direct approximation of the underlying physical viscosity, as outlined in (2a), requires very small mesh sizes (the order of the viscosity coefficient) for a non-oscillatory resolution of discontinuities [8]. Hence, we need suitable numerical diffusion operators to mimic the action of the physical viscosity.

This strategy is illustrated for two model systems in this paper. The first was explored by Abgrall and Karni in [2] and contains most of the difficulties that one might encounter in more complicated non-conservative systems. The second system is the isothermal Euler equations as described by Karni in [12].

The rest of the paper is organized as follows: in Section 2, we introduce the model system of [2] and state the associated entropy formulation. Entropy conservative schemes are described in Section 3. Physical viscosity and numerical diffusion operators are considered in Sections 4 and 5, respectively. The similar case of the isothermal Euler equations is briefly described in Section 6 .

\section{LAGRANGIAN EULER EQUATIONS}

\subsection{Conservative form}

Following [2], we consider compressible inviscid gas dynamics in Lagrangian coordinates:

$$
\begin{aligned}
v_{t}-u_{x} & =0 \\
u_{t}+p_{x} & =0 \\
E_{t}+(p u)_{x} & =0 .
\end{aligned}
$$

This is an instance of the conservation law (1.1) with $\mathbf{u}=(v, u, E)$. Here, $v$ is the specific volume, $u$ is the velocity and $p$ is the gas pressure. The spatial coordinate is mass, and the total energy is given by

$$
E=e+\frac{u^{2}}{2}
$$

with the internal energy $e$ being given by the equation of state for ideal gases,

$$
e=\frac{p v}{\gamma-1}
$$

In all computations we use a gas constant of $\gamma=1.4$. 
The system is strictly hyperbolic with eigenvalues given by 0 and $\pm c$, with $c$ being the sound speed

$$
c=\sqrt{\frac{\gamma p}{v}}
$$

The system is also equipped with an entropy function $S=\frac{-p v^{\gamma}}{\gamma-1}$. It is straightforward to check that smooth solutions of (2.1) satisfy the entropy identity

$$
S_{t} \equiv 0 \cdot
$$

The above entropy identity has to be replaced with an entropy inequality at shocks.

\subsection{Non-conservative form}

The conservative system $(2.1)$ can also be written in non-conservative form by setting $\mathbf{w}=(v, u, e)$ as the vector of unknowns. The resulting equations are

$$
\begin{array}{r}
v_{t}-u_{x}=0 \\
u_{t}+p_{x}=0 \\
e_{t}+p u_{x}=0 .
\end{array}
$$

Note that (2.5) and (2.1) are equivalent for smooth solutions. The above system can be written in the generic form (1.4) by defining

$$
\mathbf{A}=\left[\begin{array}{ccc}
0 & -1 & 0 \\
-\frac{p}{v} & 0 & \frac{\gamma-1}{v} \\
0 & p & 0
\end{array}\right]
$$

The eigenvalues of $\mathbf{A}$ are 0 and $\pm c$, as for the conservative formulation. Furthermore, smooth solutions of (2.5) satisfy the entropy identity (2.4). Observe that the entropy function $S$ can be written as a function of $v$ and $e$ alone, and hence is not strictly convex with respect to $\mathbf{w}=(v, u, e)$.

We remark that the nonconservative formulation (1.4) can be obtained from the conservative one (1.1) by multiplying the latter by the change-of-variables matrix $\mathbf{T}:=\nabla_{\mathbf{u}} \mathbf{w}(\mathbf{u})$, which in the case of Lagrangian Euler equations is

$$
\mathbf{T}=\left[\begin{array}{ccc}
1 & 0 & 0 \\
0 & 1 & 0 \\
0 & -u & 1
\end{array}\right] .
$$

\subsection{Definition of the non-conservative product}

The non-conservative system (2.5) involves a single non-conservative product $p u_{x}$. As the conservative and non-conservative forms for Lagrangian gas dynamics are formally equivalent, it is natural to select a path for the non-conservative product such that the resulting generalized Rankine-Hugoniot relations (1.5) are equivalent to the standard Rankine-Hugoniot condition (1.6) for (2.1). In [2], the authors chose the linear path

$$
\begin{aligned}
& v(s)=v_{L}+s\left(v_{R}-v_{L}\right) \\
& u(s)=u_{L}+s\left(u_{R}-u_{L}\right) \\
& p(s)=p_{L}+s\left(p_{R}-p_{L}\right) .
\end{aligned}
$$

Substituting the above choice of path in (1.5) leads to the following jump conditions across a discontinuity moving with speed $\sigma$ :

$$
\begin{aligned}
& \sigma \llbracket v \rrbracket=-\llbracket u \rrbracket \\
& \sigma \llbracket u \rrbracket=\llbracket p \rrbracket \\
& \sigma \llbracket e \rrbracket=\bar{p} \llbracket u \rrbracket,
\end{aligned}
$$


where we use the standard notation

$$
\bar{u}=\frac{u_{L}+u_{R}}{2}, \quad \llbracket u \rrbracket=u_{R}-u_{L} .
$$

Note that the above jump conditions coincide with the exact jump conditions for the conservative system (2.1). This equivalence of jump conditions provides a justification for the choice of the linear path.

\section{ENTROPY CONSERVATIVE SCHEMES}

Once the path is chosen and the corresponding solutions are defined, the aim is to design numerical schemes that approximate the correct solution of the non-conservative system (2.5). As Abgrall and Karni have shown in [2], this task is highly non-trivial and path-consistent schemes of the form (1.8) might not converge to the correct solution defined by the linear path (2.7). In fact, the authors of [2] did not describe any scheme that converged to the correct solution of (2.5). We attempt to design such convergent schemes in this paper. As stated before, the first step of our design paradigm involves the construction of entropy conservative schemes.

\subsection{Conservative systems}

We consider the semi-discrete finite volume scheme

$$
\frac{\mathrm{d}}{\mathrm{d} t} \mathbf{u}_{j}+\frac{1}{\Delta x}\left(\mathbf{F}_{j+1 / 2}^{*}-\mathbf{F}_{j-1 / 2}^{*}\right)=0
$$

with $\mathbf{F}_{j+1 / 2}^{*}=\mathbf{F}^{*}\left(\mathbf{u}_{j}, \mathbf{u}_{j+1}\right)$. (Again, time dependence is suppressed for notational convenience, and the time discretization is done with a second-order Runge-Kutta method.) Following Tadmor [22], this finite volume scheme is entropy conservative if computed solutions $\mathbf{u}_{j}$ satisfy a discrete entropy equality

$$
\frac{\mathrm{d}}{\mathrm{d} t} S\left(\mathbf{u}_{j}\right)+\frac{1}{\Delta x}\left(Q_{j+1 / 2}-Q_{j-1 / 2}\right)=0
$$

for some numerical entropy flux $Q_{j+1 / 2}=Q\left(\mathbf{u}_{j}, \mathbf{u}_{j+1}\right)$. This is guaranteed if the numerical flux function $\mathbf{F}_{j+1 / 2}^{*}$ satisfies

$$
\left\langle\llbracket \mathbf{v} \rrbracket_{j+1 / 2}, \mathbf{F}_{j+1 / 2}^{*}\right\rangle=\llbracket \Psi \rrbracket_{j+1 / 2} \quad \forall j,
$$

see [22]. Here $\mathbf{v}:=\nabla_{\mathbf{u}} S(\mathbf{u})$ are the entropy variables in (1.2) and $\Psi=\langle\mathbf{v}, \mathbf{F}\rangle-Q$ is the entropy potential.

For Lagrangian gas dynamics with entropy $S$, the entropy flux function is $Q \equiv 0$, and the entropy equality (3.2) states that entropy is constant in time. The entropy variables and potential are given by

$$
\mathbf{v}=v^{\gamma-1}\left[\begin{array}{c}
-p \\
u \\
-1
\end{array}\right], \quad \Psi=p u v^{\gamma-1} .
$$

By directly inserting $\mathbf{v}, \Psi$ into (3.3), one can obtain the following explicit expression for $\mathbf{F}^{*}$ :

$$
\mathbf{F}^{*}\left(\mathbf{u}_{L}, \mathbf{u}_{R}\right)=\left[\begin{array}{c}
-\bar{u} \\
\bar{p} \\
2 \bar{p} \bar{u}-\overline{p u}
\end{array}\right]=\left[\begin{array}{c}
-\bar{u} \\
\bar{p} \\
\frac{p_{L} u_{R}+p_{R} u_{L}}{2}
\end{array}\right] .
$$

See [8] for more information about this technique. Note that the third component of the numerical flux is not an arithmetic average of the exact fluxes of the left and right states. Inserting (3.5) into (3.1) gives the scheme

$$
\begin{aligned}
\frac{\mathrm{d}}{\mathrm{d} t} v_{j}-\frac{u_{j+1}-u_{j-1}}{2 \Delta x} & =0 \\
\frac{\mathrm{d}}{\mathrm{d} t} u_{j}+\frac{p_{j+1}-p_{j-1}}{2 \Delta x} & =0 \\
\frac{\mathrm{d}}{\mathrm{d} t} E_{j}+p_{j} \frac{u_{j+1}-u_{j-1}}{2 \Delta x}+u_{j} \frac{p_{j+1}-p_{j-1}}{2 \Delta x} & =0 .
\end{aligned}
$$


Proposition 3.1. The entropy conservative scheme (3.6) is a consistent discretization of the conservative system (2.1) and is second-order accurate. Furthermore, it satisfies the discrete entropy identity

$$
\frac{\mathrm{d}}{\mathrm{d} t} S_{j}(t)=0 \quad \forall j
$$

where $S_{j}(t)=S\left(\mathbf{u}_{j}(t)\right)=-\frac{p_{j}(t) v_{j}^{\gamma}(t)}{\gamma-1}$.

Proof. Consistency and accuracy follows from the definition. The entropy identity (3.7) is obtained by taking the inner product of $(3.6)$ with $\mathbf{v}_{j}:=\nabla_{\mathbf{u}} S\left(\mathbf{u}_{j}\right)$.

Higher order of spatial accuracy can be obtained by using the procedure of [16]. As an example, a fourth order accurate entropy conservative scheme consists of replacing $\mathbf{F}^{*}$ in (3.1) with

$$
\mathbf{F}_{j+1 / 2}^{4, *}=\frac{4}{3} \mathbf{F}^{*}\left(\mathbf{u}_{j}, \mathbf{u}_{j+1}\right)-\frac{1}{6}\left(\mathbf{F}^{*}\left(\mathbf{u}_{j-1}, \mathbf{u}_{j+1}\right)+\mathbf{F}^{*}\left(\mathbf{u}_{j}, \mathbf{u}_{j+2}\right)\right) .
$$

\subsection{Non-conservative system}

There is no general theory characterizing entropy conservative schemes for non-conservative systems like (1.4). However, we can derive an entropy conservative scheme for the model system (2.5) based on the formal equivalence between it and the conservative system (2.1). Mimicking this equivalence at the discrete level, we multiply (3.6) by the change-of-variables matrix $\mathbf{T}_{j}=\nabla_{\mathbf{u}} \mathbf{w}\left(\mathbf{u}_{j}\right)$ to obtain the following semi-discrete scheme:

$$
\begin{aligned}
\frac{\mathrm{d}}{\mathrm{d} t} v_{j}-\frac{u_{j+1}-u_{j-1}}{2 \Delta x} & =0 \\
\frac{\mathrm{d}}{\mathrm{d} t} u_{j}+\frac{p_{j+1}-p_{j-1}}{2 \Delta x} & =0 \\
\frac{\mathrm{d}}{\mathrm{d} t} e_{j}+p_{j} \frac{u_{j+1}-u_{j-1}}{2 \Delta x} & =0 .
\end{aligned}
$$

This scheme has the following properties.

Proposition 3.2. The semi-discrete scheme (3.9) is a consistent approximation of the non-conservative system (2.5) and is second-order accurate. Furthermore, it satisfies the discrete entropy identity (3.7). Hence, (3.9) is entropy conservative.

Proof. The proof of second-order accuracy follows from a Taylor expansion. To prove (3.7), we take the inner product of (3.9) by $\nabla_{\mathbf{w}} S\left(\mathbf{w}_{j}\right)$. Since

$$
\nabla_{\mathbf{w}} S(\mathbf{w})=\mathbf{T}^{-\top} \nabla_{\mathbf{u}} S(\mathbf{u})=\mathbf{T}^{-\top} \mathbf{v}
$$

the result follows from Proposition 3.1.

A fourth-order entropy conservative version of (3.9), obtained by multiplying (3.8) by $\mathbf{T}_{j}=\nabla_{\mathbf{u}} \mathbf{w}\left(\mathbf{u}_{j}\right)$, is given by

$$
\begin{aligned}
& \frac{\mathrm{d}}{\mathrm{d} t} v_{j}(t)-\frac{1}{\Delta x}\left(\frac{2}{3}\left(u_{j+1}-u_{j-1}\right)-\frac{1}{12}\left(u_{j+2}-u_{j-2}\right)\right)=0 \\
& \frac{\mathrm{d}}{\mathrm{d} t} u_{j}(t)+\frac{1}{\Delta x}\left(\frac{2}{3}\left(p_{j+1}-p_{j-1}\right)-\frac{1}{12}\left(p_{j+2}-p_{j-2}\right)\right)=0 \\
& \frac{\mathrm{d}}{\mathrm{d} t} e_{j}(t)+\frac{p_{j}}{\Delta x}\left(\frac{2}{3}\left(u_{j+1}-u_{j-1}\right)-\frac{1}{12}\left(u_{j+2}-u_{j-2}\right)\right)=0 .
\end{aligned}
$$




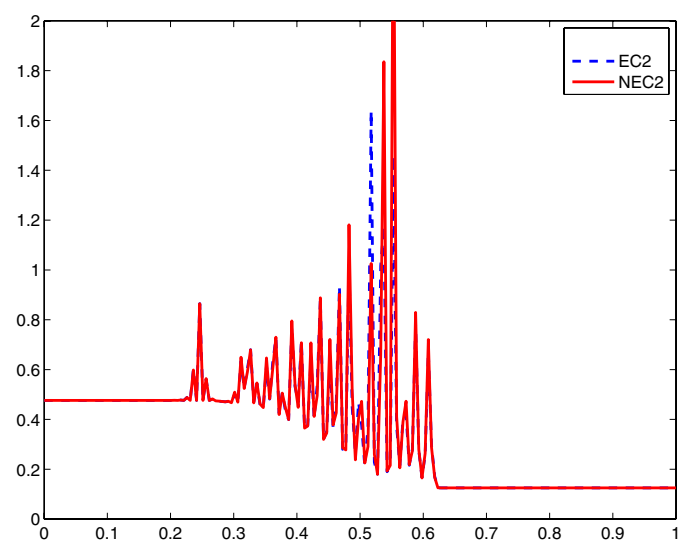

Figure 1. Computed density for the Riemann problem (3.12) with the EC2 and NEC2 schemes at time $t=0.25$ on a mesh with 200 points.

\subsection{Numerical experiments}

In this section we test the two second-order accurate, entropy conservative schemes

EC2: The conservative scheme (3.6).

NEC2: The non-conservative scheme (3.9).

Following [2], we consider a Riemann problem whose solution is a single right-going shock. Fixing the right state $\mathbf{u}_{R}$, we can use the generalized Rankine-Hugoniot condition (2.8) to express the left state variables $v_{L}, u_{L}$ as a function of $p_{L}$ :

$$
v_{L}=\frac{2 \gamma \bar{p}+\llbracket p \rrbracket}{2 \gamma \bar{p}-\llbracket p \rrbracket} v_{R}, \quad u_{L}=u_{R}+\sqrt{\frac{2 v_{R} \llbracket p \rrbracket^{2}}{2 \gamma \bar{p}-\llbracket p \rrbracket}} .
$$

Setting

$$
p_{L}=1, \quad\left(v_{R}, u_{R}, p_{R}\right)=(8,0,0.1),
$$

we obtain $v_{L} \approx 2.0984, u_{L} \approx 2.3047$. The shock moves at a speed $\sigma \approx 0.3905$. The initial data is set to be

$$
\mathbf{u}_{0}(x)=\left\{\begin{array}{lll}
\mathbf{u}_{L} & \text { if } & x<0.5 \\
\mathbf{u}_{R} & \text { if } & x>0.5
\end{array}\right.
$$

and we compute for $x \in[0,1]$ with non-reflecting boundary conditions. The domain is partitioned into 200 grid cells. In Figure 1 we plot the density $(1 / v)$ at time $t=0.25$ computed with the EC2 scheme for the conservative system (2.1) and with the NEC2 scheme for the non-conservative system (2.5). Both schemes approximate the single shock solution qualitatively. As expected, there are large oscillations behind the shock as both schemes lack any dissipation mechanism. We introduce dissipation mechanisms in subsequent sections. We note that there are very minor differences between the entropy conservative schemes approximating the conservative system (2.1) and the non-conservative system (2.5).

\section{Physical Diffusion}

In this section, we introduce explicit physical diffusion for both the conservative (2.1) and nonconservative (2.5) systems in order to model the dissipation at shocks. We use two different sets of explicit diffusion operators. 


\subsection{Uniform diffusion}

\section{Conservative form}

We add explicit uniform diffusion to the conservative system (2.1) by introducing a Laplacian on the righthand side of (2.1) to obtain

$$
\begin{aligned}
v_{t}-u_{x} & =\mu v_{x x} \\
u_{t}+p_{x} & =\mu u_{x x} \\
E_{t}+(p u)_{x} & =\mu E_{x x} .
\end{aligned}
$$

Here, $\mu$ is the diffusion coefficient. Formally, the solution $\mathbf{u}^{\mu}=(v, u, E)$ of (4.1) converges to the entropy solution $\mathbf{u}$ of $(2.1)$ as $\mu \rightarrow 0$.

Non-conservative form

By multiplying (4.1) by $\mathbf{T}$ we can derive the equivalent version of (4.1) for the non-conservative system (2.5) as

$$
\begin{aligned}
v_{t}-u_{x} & =\mu v_{x x} \\
u_{t}+p_{x} & =\mu u_{x x} \\
e_{t}+p u_{x} & =\mu e_{x x}+\mu u_{x}^{2} .
\end{aligned}
$$

An alternative diffusion operator for the non-conservative system (2.5) would consist of adding a Laplacian to it, leading to the system

$$
\begin{aligned}
v_{t}-u_{x} & =\mu v_{x x} \\
u_{t}+p_{x} & =\mu u_{x x} \\
e_{t}+p u_{x} & =\mu e_{x x} .
\end{aligned}
$$

Note that the only difference between these two formulations is the $\mu u_{x}^{2}$ term in (4.2).

\subsection{Navier-Stokes viscosity}

A more physically relevant diffusion operator for Lagrangian gas dynamics is provided by the Navier-Stokes type viscosity term.

Conservative form

The Navier-Stokes equations in Lagrangian coordinates are given by

$$
\begin{aligned}
v_{t}-u_{x} & =0 \\
u_{t}+p_{x} & =\mu\left(\frac{u_{x x}}{v}-\frac{u_{x} v_{x}}{v^{2}}\right) \\
E_{t}+(p u)_{x} & =\mu\left(\frac{u_{x}^{2}}{2 v}\right)_{x},
\end{aligned}
$$

where $\mu$ is the viscosity coefficient [18].

\section{Non-conservative form}

The corresponding Navier-Stokes equations for the non-conservative system (2.5) are again obtained by multiplying (4.4) by $\mathbf{T}$, obtaining

$$
\begin{aligned}
v_{t}-u_{x} & =0 \\
u_{t}+p_{x} & =\mu\left(\frac{u_{x x}}{v}-\frac{u_{x} v_{x}}{v^{2}}\right) \\
e_{t}+p u_{x} & =\mu \frac{u_{x}^{2}}{v} .
\end{aligned}
$$




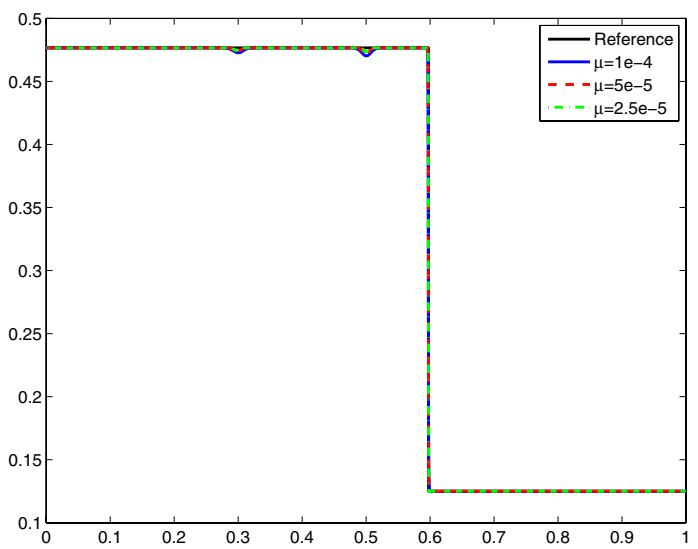

(a) Density

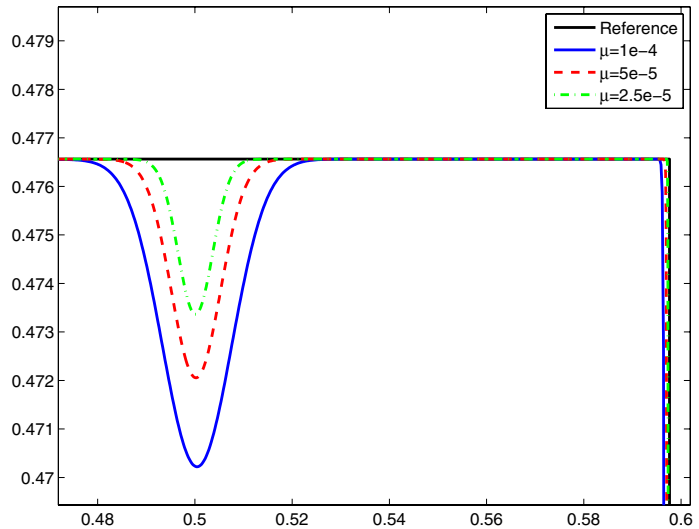

(b) Density, closeup

Figure 2. Computed solution for the Riemann problem (3.12) for the viscous equation (4.1) with a second-order scheme.

We remark that the Navier-Stokes equations in both the conservative (4.4) and non-conservative (4.5) forms satisfy the entropy inequality

$$
S_{t} \leq 0
$$

\subsection{Numerical schemes}

The left hand sides of all the above convection-diffusion equations is either the conservative system (2.1) or the non-conservative system (2.5), and to discretize them we use the entropy conservative schemes of the previous section. The right hand side consists of explicit diffusion operators and we discretize them by standard central finite difference operators. For second order discretization, we use

$$
\begin{aligned}
g_{x} & \approx \frac{g_{j+1}-g_{j-1}}{2 \Delta x} \\
g_{x x} & \approx \frac{g_{j+1}-2 g_{j}+g_{j-1}}{\Delta x^{2}},
\end{aligned}
$$

and for fourth-order accuracy, we use

$$
\begin{aligned}
g_{x} & \approx \frac{1}{\Delta x}\left(\frac{2}{3}\left(g_{j+1}-g_{j-1}\right)-\frac{1}{12}\left(g_{j+2}-g_{j-2}\right)\right) \\
g_{x x} & \approx \frac{1}{\Delta x^{2}}\left(\frac{4}{3}\left(g_{j+1}+g_{j-1}\right)-\frac{5}{2} g_{j}-\frac{1}{12}\left(g_{j+2}+g_{j-2}\right)\right) .
\end{aligned}
$$

Here $g$ is any given function and $g_{j}=g\left(x_{j}\right)$.

\subsection{Numerical experiments}

We consider the Riemann problem (3.12) for four different convection-diffusion equations: (4.1) for the conservative system and (4.2), (4.3) and (4.5) for the non-conservative system. The aim is to ascertain the viscous profile corresponding to very small viscosity coefficients for both systems with different regularizations.

Figure 2 shows density for the system (4.1) along with the exact single-shock solution. We use viscosity coefficients $\mu=10^{-4}, 0.5 \times 10^{-4}$ and $0.25 \times 10^{-4}$; to avoid oscillations in the solution we have to use a high number of mesh points: 4000, 8000 and 16000 , respectively. Increasing the number of mesh points further for 


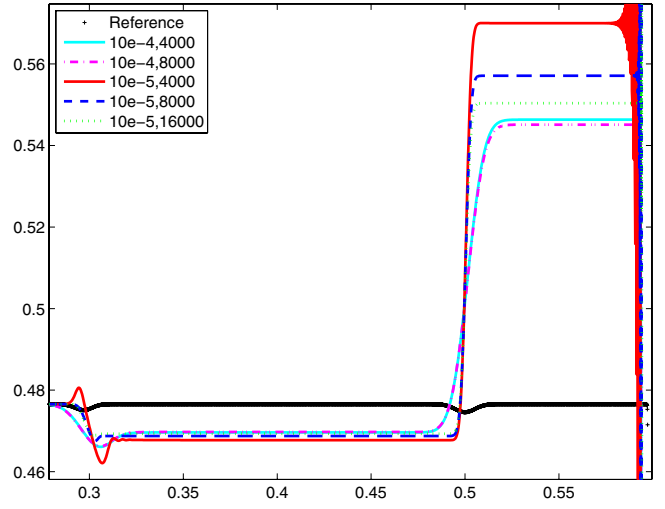

(a) Second order, (4.3)

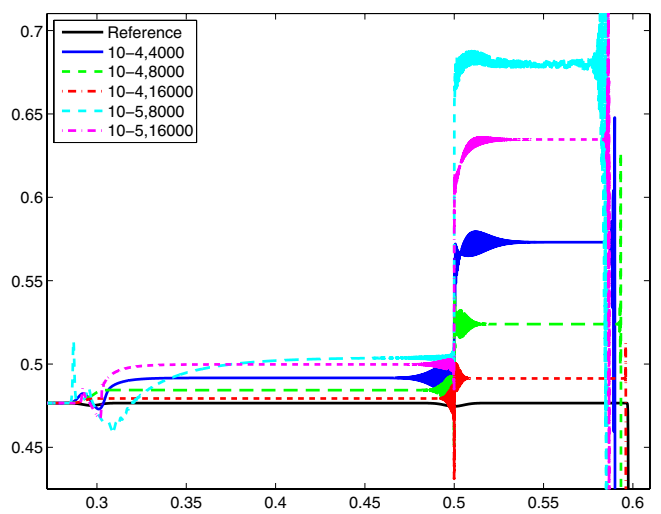

(c) Second order, (4.5)

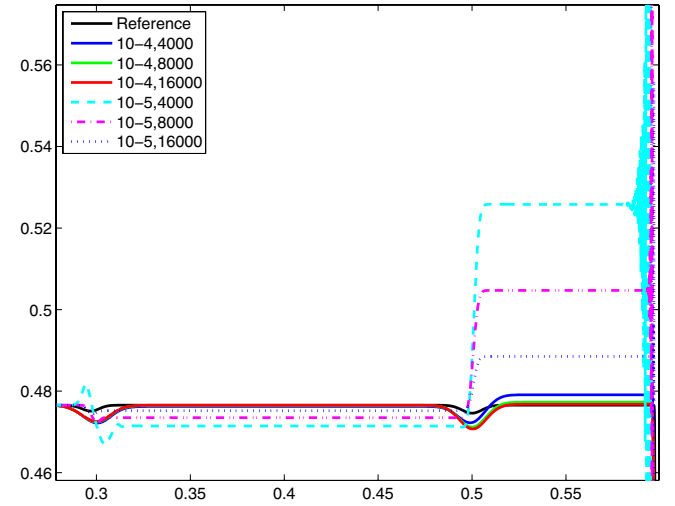

(b) Second order, (4.2)

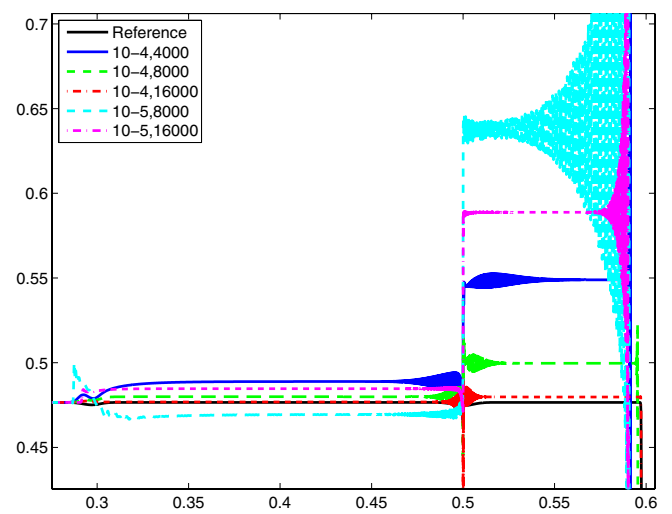

(d) Fourth order, (4.5)

FIgURE 3. Computed density for (3.12) with different regularizations of the non-conservative system (2.5). In each plot, the value of the viscosity coefficient and the number of mesh points are indicated.

fixed $\mu$ does not change the solution much. It is clear that as $\mu$ decreases, the computed solutions approach the solution of the inviscid conservative system (2.1). What is more, the discontinuity is propagated at the correct speed in all three simulations. Similar results (not shown here) were obtained for the Navier-Stokes regularization (4.4) of the conservative system (2.1).

The same experiment is repeated for the diffusive regularizations of the non-conservative system (2.5) and the computed densities are shown in Figure 3. There are two main differences between the results for the conservative and non-conservative cases: although the viscous profiles for (4.2) and (4.5) converge to the single shock solution in the inviscid case, the rate of convergence with respect to the mesh size (for a fixed $\mu$ ) is very slow, particularly for the Navier-Stokes regularization (4.5). Furthermore, the rate of convergence decreases when the coefficient $\mu$ is reduced. Some deterioration in the rate may be due to oscillations on account of inadequate resolution. This convergence is accelerated when fourth-order discretizations are used, as illustrated in Figure 3d (contrast with Fig. 3c).

The second major difference between the two cases is that the viscous profile corresponding to the discrete Laplace operator (4.3) does not converge to the inviscid solution. Instead, it converges to a solution with a 
higher intermediate density. This leads to the conclusion that the limit of viscous regularizations for the nonconservative system (2.5) is sensitive to the choice of the diffusion operator.

\section{NumERICAL DIFFUSION}

The addition of explicit viscosity to the entropy conservative schemes is problematic as the viscosity coefficients have to be very small in order to approximate the viscous limit, as was demonstrated in the previous section. Small viscosity coefficients require very fine meshes (mesh size being on the order of the viscosity coefficients) for resolving the solution without unphysical oscillations. Such fine meshes are not computationally feasible, especially for multi-dimensional problems. Hence, we need to devise suitable numerical diffusion operators that mimic the effects of physical viscosity while capturing shocks without unphysical oscillations.

\subsection{Conservative form}

Any standard entropy stable diffusion operator can be added to the entropy conservative scheme (3.6) (see [23]). For simplicity, we choose a Lax-Friedrichs type operator:

$$
\mathbf{P}_{j+1 / 2}=\frac{c_{\max }}{2 \Delta x}\left(\mathbf{u}_{j+1}-\mathbf{u}_{j}\right) .
$$

Here,

$$
c_{\max }=\max _{j}\left|c_{j}\right|,
$$

with $c_{j}$ being the sound speed corresponding to the state $\mathbf{u}_{j}$ at some time $t$. The resulting (semi-discrete) scheme is

$$
\frac{\mathrm{d}}{\mathrm{d} t} \mathbf{u}_{j}=-\frac{1}{\Delta x}\left(\mathbf{F}_{j+1 / 2}^{*}-\mathbf{F}_{j-1 / 2}^{*}\right)+\Delta t\left(\mathbf{P}_{j+1 / 2}-\mathbf{P}_{j-1 / 2}\right)
$$

Here, $\mathbf{F}^{*}$ is the entropy conservative flux defined in (3.5). The diffusion operator in (5.2) corresponds to setting the viscosity coefficient in (4.1) as $\mu=\frac{c_{\max } \Delta x}{2}$, which vanishes as the mesh is refined. A less dissipative choice would be to scale the dissipation with local wave speeds, which gives a Rusanov-type scheme.

\subsection{Non-conservative form}

Analogous to the conservative case, we can introduce numerical diffusion operators for the non-conservative system (2.5) by replacing the viscosity coefficients in front of the regularizations (4.2), (4.3) and (4.5) by a scaled version of the mesh size. In particular, we select $\mu=\frac{c_{\max } \Delta x}{2}$, as above. The left-hand sides are discretized using the entropy conservative scheme (3.9). This leads to the following schemes:

\section{Discrete Laplacian}

Discretizing the Laplacian-type diffusion operator in (4.3) gives the following Lax-Friedrichs-type scheme:

$$
\begin{aligned}
\frac{\mathrm{d}}{\mathrm{d} t} v_{j}-\frac{1}{2 \Delta x}\left(u_{j+1}-u_{j-1}\right) & =\frac{c_{\max }}{2 \Delta x}\left(v_{j+1}-2 v_{j}+v_{j-1}\right) \\
\frac{\mathrm{d}}{\mathrm{d} t} u_{j}+\frac{1}{2 \Delta x}\left(p_{j+1}-p_{j-1}\right) & =\frac{c_{\max }}{2 \Delta x}\left(u_{j+1}-2 u_{j}+u_{j-1}\right) \\
\frac{\mathrm{d}}{\mathrm{d} t} e_{j}+\frac{p_{j}}{2 \Delta x}\left(u_{j+1}-u_{j-1}\right) & =\frac{c_{\max }}{2 \Delta x}\left(e_{j+1}-2 e_{j}+e_{j-1}\right) .
\end{aligned}
$$




\section{Modified discrete Laplacian}

Instead of rescaling the regularized system (4.3), we can rescale the regularized system (4.2) (derived from the underlying system (4.1)) resulting in the following modified Lax-Friedrichs type scheme:

$$
\begin{aligned}
\frac{\mathrm{d}}{\mathrm{d} t} v_{j}-\frac{1}{2 \Delta x}\left(u_{j+1}-u_{j-1}\right)= & \frac{c_{\max }}{2 \Delta x}\left(v_{j+1}-2 v_{j}+v_{j-1}\right) \\
\frac{\mathrm{d}}{\mathrm{d} t} u_{j}+\frac{1}{2 \Delta x}\left(p_{j+1}-p_{j-1}\right)= & \frac{c_{\max }}{2 \Delta x}\left(u_{j+1}-2 u_{j}+u_{j-1}\right) \\
\frac{\mathrm{d}}{\mathrm{d} t} e_{j}+\frac{p_{j}}{2 \Delta x}\left(u_{j+1}-u_{j-1}\right)= & \frac{c_{\max }}{2 \Delta x}\left(e_{j+1}-2 e_{j}+e_{j-1}\right) \\
& +\frac{c_{\max }}{2 \Delta x}\left(\frac{u_{j+1}-u_{j-1}}{2}\right)^{2} .
\end{aligned}
$$

Note that the only difference between (5.3) and (5.4) is the term approximating $\mu u_{x}^{2}$ in the third component.

\section{Discrete Navier-Stokes operator}

A discretization of the Navier-Stokes viscosity in (4.5) results in the scheme

$$
\begin{aligned}
\frac{\mathrm{d}}{\mathrm{d} t} v_{j}-\frac{1}{2 \Delta x}\left(u_{j+1}-u_{j-1}\right)= & \frac{c_{\max }}{2 \Delta x}\left(v_{j+1}-2 v_{j}+v_{j-1}\right) \\
\frac{\mathrm{d}}{\mathrm{d} t} u_{j}+\frac{1}{2 \Delta x}\left(p_{j+1}-p_{j-1}\right)= & \frac{c_{\max }}{2 \Delta x v_{j}}\left(u_{j+1}-2 u_{j}+u_{j-1}\right) \\
& -\frac{c_{\max }}{8 \Delta x\left(v_{j}\right)^{2}}\left(u_{j+1}-u_{j-1}\right)\left(v_{j+1}-v_{j-1}\right) \\
\frac{\mathrm{d}}{\mathrm{d} t} e_{j}+\frac{p_{j}}{2 \Delta x}\left(u_{j+1}-u_{j-1}\right)= & \frac{c_{\max }}{2 \Delta x v_{j}}\left(\frac{u_{j+1}-u_{j-1}}{2}\right)^{2} .
\end{aligned}
$$

Note that this scheme is not strictly a discrete version of the Navier-Stokes equation (4.5) as we have added numerical diffusion in the density equation.

Remark 5.1. All of the aforementioned schemes are first-order accurate. An alternative would be to add fourthorder diffusion operators (4.8) to the fourth-order accurate entropy conservative scheme (3.10). We consider such schemes in the next section. However, the schemes are still first-order accurate as the diffusion coefficient is proportional to the mesh size.

\subsection{Numerical experiments}

In this section, we test the following first-order accurate schemes:

ECS: (5.2) solving the conservative system (2.1).

ELF: (5.3) with Lax-Friedrichs type diffusion.

ELM: (5.4) with modified Lax-Friedrichs type diffusion.

ENS: (5.5) with Navier-Stokes type diffusion operator.

We can expect these schemes to mimic the behavior of their respective counterpart in Section 4, while at the same time introducing more diffusion, thus requiring fewer grid cells to remove oscillatory behavior.

In some experiments, we also test the fourth-order version of the above schemes and label them by ELF4, ELM4 and ENS4 respectively. 


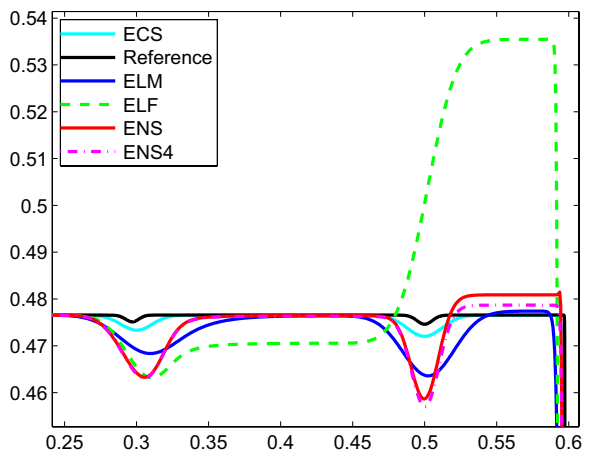

(a) Density

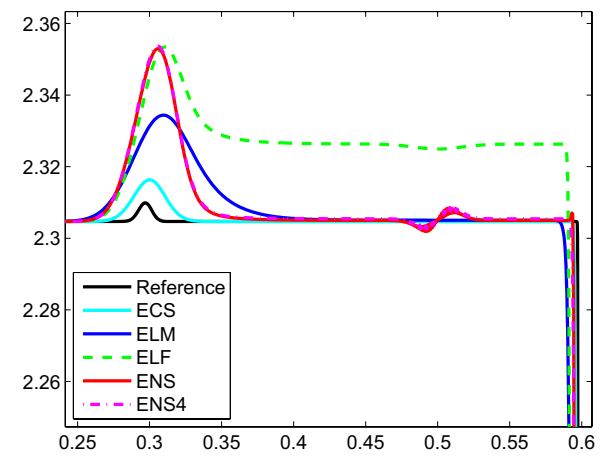

(b) Velocity

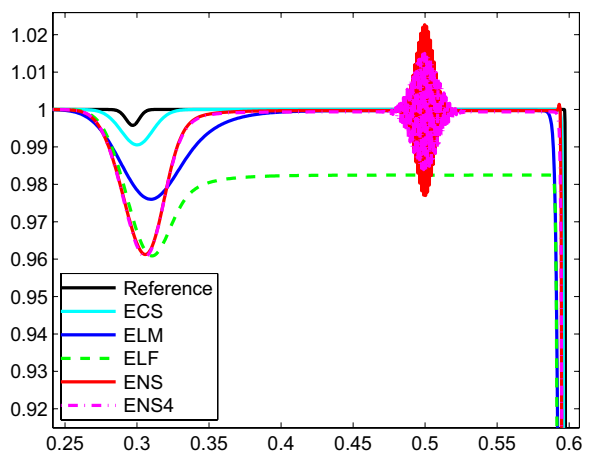

(c) Pressure

Figure 4. Computed density for (3.12) with different schemes for (2.1) and (2.5). All the results are shown at $t=0.25$ and are computed on a mesh of 1500 points. The reference solution is computed with the ECS schemes on a mesh of 8000 points.

\subsubsection{Single shock}

We repeat the initial-value problem (3.12) with the four schemes on a mesh of 1500 points. Computed density, velocity and pressure are shown in Figure 4. It is evident that the Lax-Friedrichs type ELF scheme computes wrong intermediate states in all three variables, with the largest deviations lying in the density. This is not unexpected, as using the explicit Laplacian type diffusion operator in the regularized system (4.3) led to a viscous profile that did not converge to the correct solution (see Fig. 3a). Since the ELF diffusion operator models the action of a Laplacian, it is thus not surprising that the resulting scheme converges to an incorrect solution. In fact, the results for the ELF scheme are comparable to those obtained by Abgrall and Karni (see Fig. 1 in [2]) with the path consistent Roe and Lax-Friedrichs schemes. The results suggest that the reason the path consistent schemes might not converge to the correct solution is the dynamics of the numerical diffusion operator, thus justifying the speculations of [2].

Conversely, the modified Lax-Friedrichs type ELM scheme and the Navier-Stokes based ENS scheme compute correct intermediate states, albeit with small deviations. Again, this is expected as their regularized counterparts (4.2) and (4.5) converge towards the single-shock solution.

To investigate the question of convergence further, we repeat the experiment with the ELF and ELM schemes on a sequence of meshes. The resulting density is shown in Figure 5. Apart from a slight difference directly 


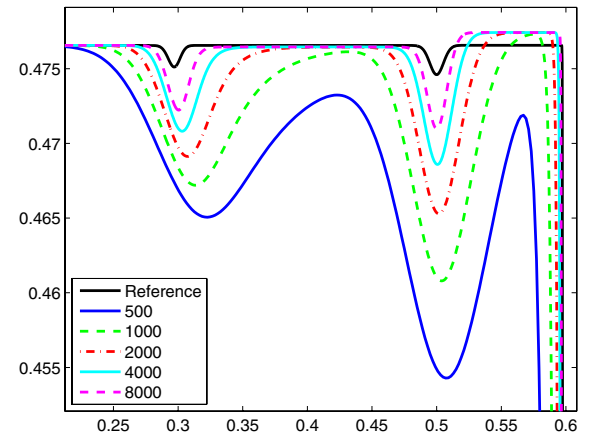

(a) ELM scheme

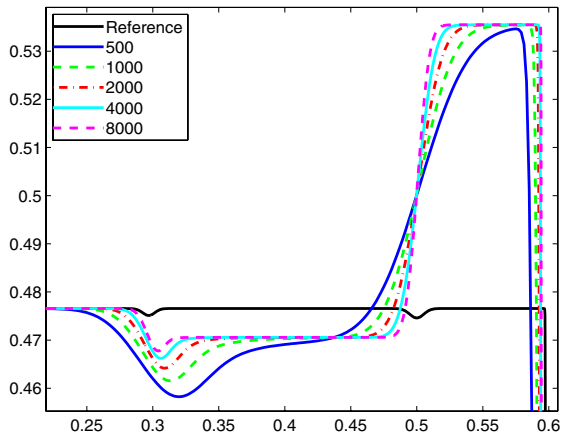

(b) ELF scheme

Figure 5. Density for (3.12), computed at time $t=0.25$ on a sequence of meshes with the ELF and ELM schemes. Note the different scaling of the $y$-axis.

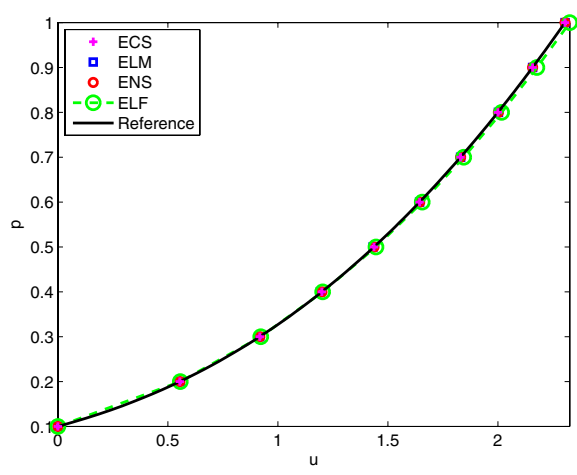

(a) Hugoniot locus

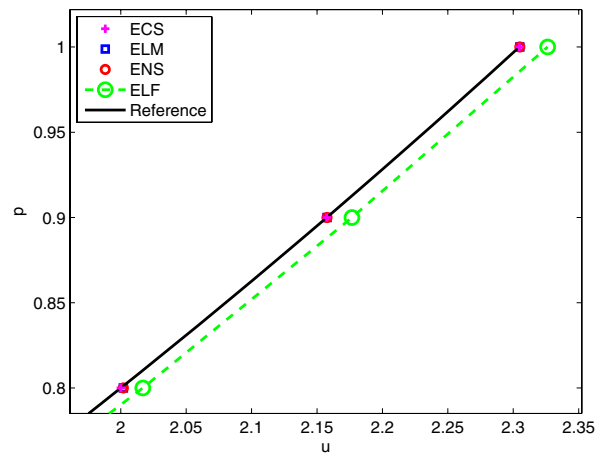

(b) Closeup

Figure 6. The numerical Hugoniot locus in the $u$ - $p$ plane computed with the ECS, ELF, ELM and ENS schemes.

behind the shock, the ELM scheme displays a clear, monotonous convergence towards the reference solution. The ELF scheme also shows convergence, but towards an entirely unphysical solution.

\subsubsection{Hugoniot locus}

Following $[2,5]$, we compute the Hugoniot locus of the ECS, ELF, ELM and ENS schemes. This is done by fixing $\left(v_{R}, u_{R}, p_{R}\right)=(8,0,0.1)$ (as before) while varying $p_{L}$ between 0.2 and 1 . For each value of $p_{L}$, the corresponding single-shock left state is calculated from (3.11) and the initial-value problem (3.12) is solved with the four schemes. The computed value directly behind the shock is plotted in the $u-p$ plane in Figure 6 . We see that the ECS, ELF and ENS schemes approximate the exact Hugoniot locus very accurately, whereas the ELF scheme converges to a different Hugoniot locus. This indicates that choosing a suitable numerical diffusion operator is absolutely essential in obtaining convergence to a correct solution. 


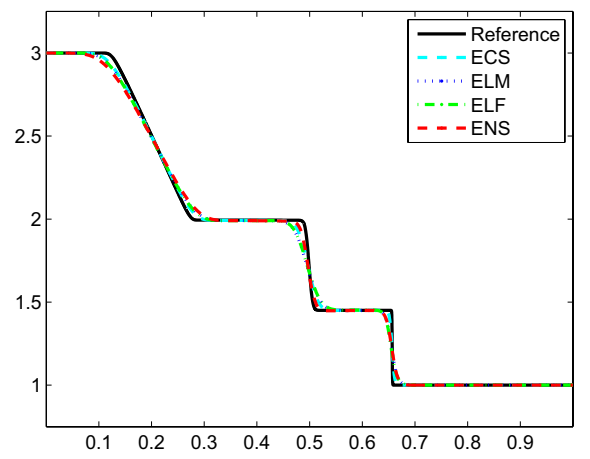

(a) Density

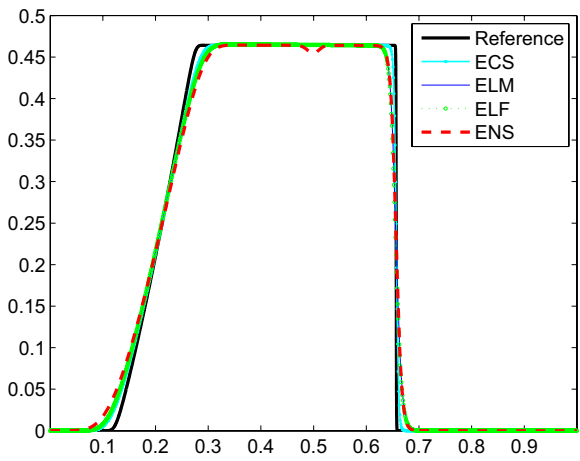

(b) Velocity

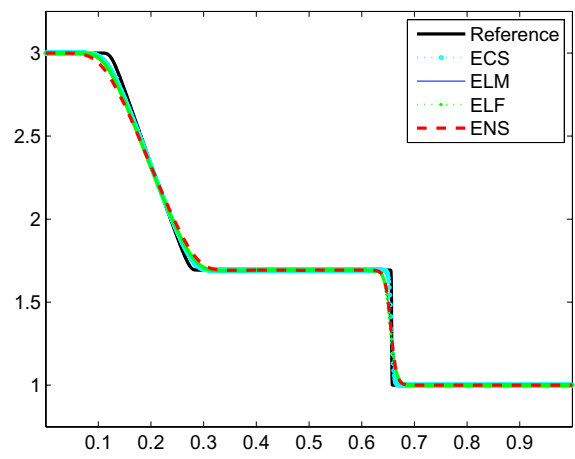

(c) Pressure

Figure 7. Computed solutions for the Sod shock tube (5.6) with different schemes for (2.1) and (2.5). All the results are shown at $t=0.105$ on a mesh of 1000 points. The reference solution is computed with the ECS schemes on a mesh of 8000 points.

\subsubsection{Sod shock tube}

We consider the Riemann problem

$$
\left(v_{0}(x), u_{0}(x), p_{0}(x)\right)=\left\{\begin{array}{lll}
(1 / 3,0,3) & \text { if } & x<0.5 \\
(1,0,1) & \text { if } & x>0.5 .
\end{array}\right.
$$

This is a variant of the standard Sod shock tube problem [17] and the exact solution consists of a left going rarefaction, a right going shock and a right going contact discontinuity. Figure 7 shows the computed density with the ECS, ELF, ELM and ENS schemes. The results indicate that that all the schemes, including the ELF scheme, converge to the correct solution in this case.

\section{Isothermal Euler equations}

To illustrate our approach in a different setting, we consider the isothermal Euler equations

$$
\begin{aligned}
\rho_{t}+(\rho u)_{x} & =0 \\
(\rho u)_{t}+\left(\rho u^{2}+c^{2} \rho\right)_{x} & =0,
\end{aligned}
$$


obtained by letting $\gamma \rightarrow 1$ in the Euler equations [6,12]. Here, $c$ is the (constant) sound speed. The system (6.1) has eigenvalues $u \pm c$ and hence is strictly hyperbolic. We set $c=1$ in the remainder.

Energy is not a conserved variable in the isothermal Euler equations; rather, it gives rise to the entropy pair

$$
S=\rho\left(\frac{u^{2}}{2}+\log \rho\right), \quad Q=\rho u\left(\frac{u^{2}}{2}+\log \rho+1\right) .
$$

We will consider the following regularization of (6.1):

$$
\begin{aligned}
\rho_{t}+(\rho u)_{x} & =\mu \rho_{x x} \\
(\rho u)_{t}+\left(\rho u^{2}+\rho\right)_{x} & =\mu(\rho u)_{x x} .
\end{aligned}
$$

Formally, the solution of (6.2) converges to the solution of (6.1) as $\mu \rightarrow 0$.

Karni [12] considers the following alternative formulation of $(6.1)$ :

$$
\begin{aligned}
\rho_{t}+(\rho u)_{x} & =0 \\
u_{t}+\left(\frac{u^{2}}{2}+\log \rho\right)_{x} & =0 .
\end{aligned}
$$

This system can be written in the nonconservative form (1.4) by letting

$$
\mathbf{w}=(\rho, u), \quad A(\mathbf{w})=\left[\begin{array}{cc}
u & \rho \\
1 / \rho & u
\end{array}\right] .
$$

We remark that although (6.3) is written in divergence form, the velocity $u$ is not a conserved variable. To see this, we keep the right-hand side terms of (6.2) to obtain the equivalent formulation

$$
\begin{aligned}
\rho_{t}+(\rho u)_{x} & =\mu \rho_{x x} \\
u_{t}+\left(\log \rho+\frac{u^{2}}{2}\right)_{x} & =\mu u_{x x}+2 \mu(\log \rho)_{x} u_{x} .
\end{aligned}
$$

Clearly the term $2 \mu(\log \rho)_{x} u_{x}$ in the equation for $u$ cannot be written in divergence form, and so even in the limit $\mu \rightarrow 0, u$ is not conserved.

A naive regularization of (6.3) would involve adding a Laplacian to the right-hand side, obtaining the system

$$
\begin{array}{r}
\rho_{t}+(\rho u)_{x}=\mu \rho_{x x} \\
u_{t}+\left(\log \rho+\frac{u^{2}}{2}\right)_{x}=\mu u_{x x} .
\end{array}
$$

As we demonstrate numerically in the next section, the solution of (6.5) does not converge to the entropy solution of $(6.1)$ as $\mu \rightarrow 0$.

\subsection{Numerical schemes}

As the inviscid system (6.3) is written in divergence form, it is straightforward to obtain entropy conservative schemes $[8,23]$. We omit the details and give its formulation:

$$
\frac{\mathrm{d}}{\mathrm{d} t} \mathbf{w}_{j}+\frac{1}{\Delta x}\left(\mathbf{F}_{j+1 / 2}^{*}-\mathbf{F}_{j-1 / 2}^{*}\right)=0, \quad \mathbf{F}_{j+1 / 2}^{*}=\left[\begin{array}{c}
\overline{\rho u} \\
\frac{u^{2}}{2}+\overline{\log \rho}
\end{array}\right] .
$$

To obtain entropy stable schemes we discretize the right-hand sides of (6.4) and (6.5) using central differences, as before. The resulting schemes are named ELM and ELF, respectively, corresponding to their counterparts in Section 5 . The viscosity coefficient is set as $\mu=\frac{c_{\max } \Delta x}{2}$, as before, where

$$
c_{\max }:=\max _{j}\left|u_{j}\right|+c
$$

is the maximum eigenvalue of the solution. 


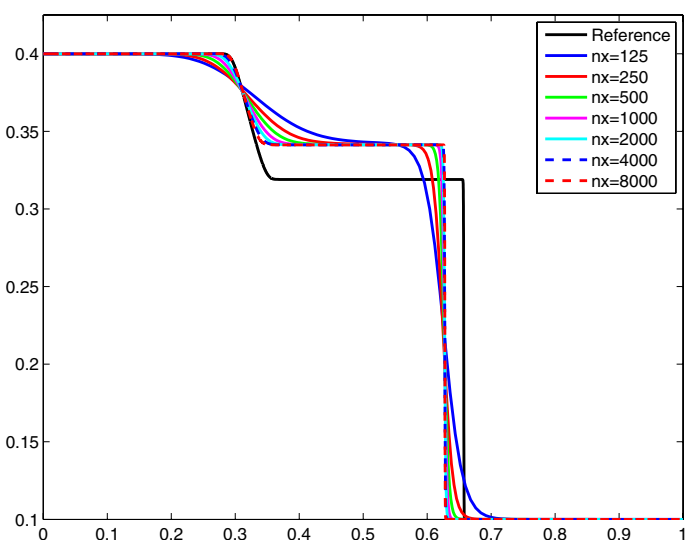

(a) Density

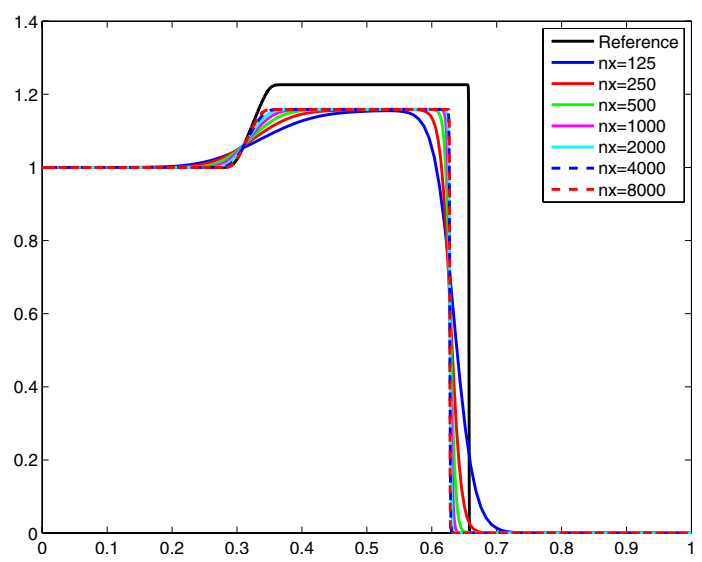

(b) Velocity

Figure 8. Solution computed with the ELF scheme.

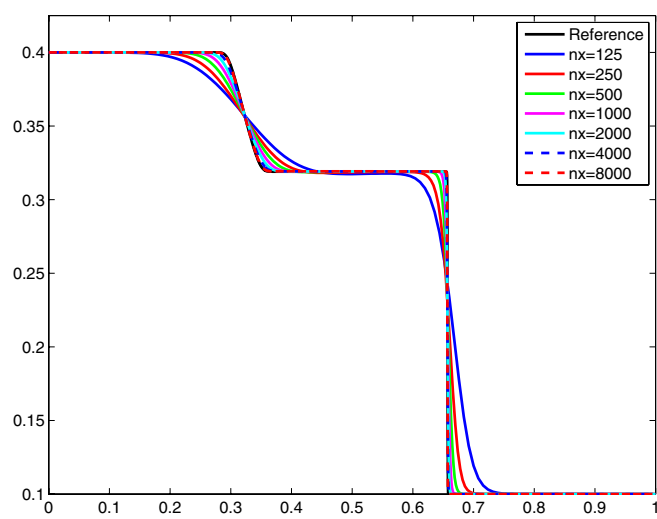

(a) Density

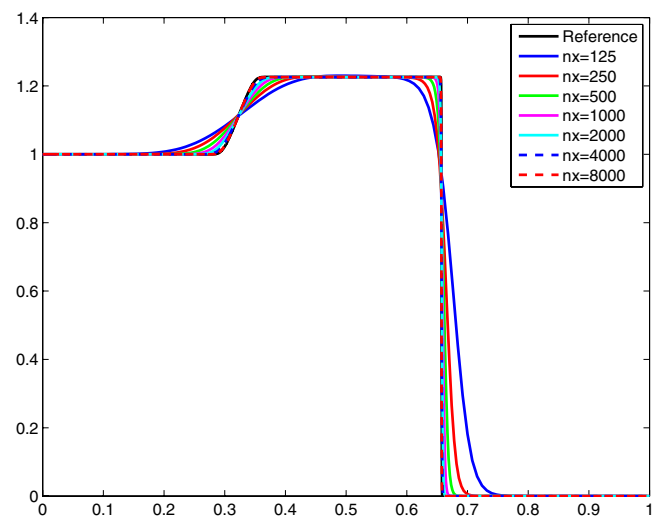

(b) Velocity

Figure 9. Solution computed with the ELM scheme.

\subsection{Numerical experiment}

We consider the following numerical experiment, taken from [12]:

$$
\left(\rho_{0}(x), u_{0}(x)\right)=\left\{\begin{array}{lll}
(0.4,1) & \text { if } & x<0.5 \\
(0.1,0) & \text { if } & x>0.5 .
\end{array}\right.
$$

We solve for $x \in[0,1]$ up to $t=0.2$. The computed solutions are plotted in Figures 8 and 9 , along with a reference solution computed with the Rusanov scheme for the conservative system (6.1). We see clearly that the ELM scheme converges to the correct solution, whereas the ELF scheme converges to an unphysical solution. This incorrect solution corresponds very well to the solutions obtained with the deficient schemes considered in [12] (see Figs. 2a and 3a of that paper). Conversely, the convergent ELM scheme corresponds well with the corrected scheme of [12].

Again we see that a faithful discretization of the viscous terms of the conservative formulation (6.2) is vital to the convergence of the nonconservative formulation. 


\section{Conclusion}

The design of efficient numerical schemes for the non-conservative hyperbolic system (1.4) is very challenging as the solution is highly dependent on the choice of a path. How to make this choice is still an unsolved problem. The available theoretical framework of path-consistent schemes [21] is inadequate $[2,5]$, the main shortcoming of this approach being the lack of convergence of path-consistent schemes to the correct solution. This was illustrated in [2] for the non-conservative version of Lagrangian gas dynamics (2.5). The correction approach in [12] seems to work for the cases considered therein, but has the disadvantage of requiring a conservative form of the system, in addition to being potentially hard to derive.

We have presented an alternative paradigm for the design of numerical schemes for non-conservative systems based on three ingredients: (i) an entropy conservative discretization; (ii) explicit discretization of physical viscosity; and (iii) the design of numerical diffusion operators that mimic the effect of the physical viscosity at any given mesh resolution.

This approach has been illustrated for the Lagrangian gas dynamics system (2.5) and for a version of the isothermal Euler equations (6.3). We demonstrate that schemes based on this design paradigm have better convergence properties. The schemes are simple to implement and computationally inexpensive. The choice of the numerical diffusion operator was absolutely crucial, as a naive choice led to convergence to incorrect solutions of the underlying conservative system.

The main drawbacks of the current approach lie in the fact that we require an entropy framework as well as explicit information about the underlying viscous mechanisms, and that the schemes have to be tailored to each individual system under consideration. However, most systems arising in physics are equipped with an entropy formulation, and explicit information about the underlying viscous operators is readily available. The construction of the entropy conservative schemes presented here may seem somewhat ad-hoc. In an upcoming paper we present a unified framework for constructing entropy conservative, path-consistent methods for any given system.

We plan to extend the current framework for multi-layer shallow water equations and for multi-phase flows in a forthcoming paper. Other directions of future research are to design high-order schemes and address problems in several space dimensions. The current approach is quite promising on both issues.

\section{REFERENCES}

[1] R. Abgrall and S. Karni, Two-layer shallow water system: a relaxation approach. SIAM. J. Sci. Comput. 31 (2009) $1603-1627$.

[2] R. Abgrall and S. Karni, A comment on the computation of non-conservative products. J. Comput. Phys. 229 (2010) $2759-2763$.

[3] E. Audusse, F. Bouchut, M.O. Bristeau, R. Klien and B. Perthame, A fast and stable well-balanced scheme with hydrostatic reconstruction for shallow water flows. SIAM. J. Sci. Comput. 25 (2004) 2050-2065.

[4] E. Audusse and M.O. Bristeau, Finite volume solvers for multi-layer Saint-Venant system. Int. J. Appl. Math. Comput. Sci. 17 (2007) 311-319.

[5] M.J. Castro, P. LeFloch, M.L. Munoz Ruiz and C. Pares, Why many theories of shock waves are necessary: Convergence error in formally path-consistent schemes. J. Comput. Phys. 227 (2008) 8107-8129.

[6] G.-Q. Chen, C. Christoforou and Y. Zhang, Continuous dependence of entropy solutions to the euler equations on the adiabatic exponent and mach number. Arch. Ration. Mech. Anal. 189 (2008) 97-130.

[7] G. Dal Maso, P. LeFloch and F. Murat, Definition and weak stability of nonconservative products. J. Math. Pures. Appl. 74 (1995) 483-548.

[8] U.S. Fjordholm, S. Mishra and E. Tadmor, Energy preserving and energy stable schemes for the shallow water equations, Foundations of Computational Mathematics, Proc. FoCM held in Hong Kong 2008, London Math. Soc. Lecture Notes Ser. 363, edited by F. Cucker, A. Pinkus and M. Todd (2009) 93-139.

[9] U.S. Fjordholm, S. Mishra and E. Tadmor, Well-balanced and energy stable schemes for the shallow water equations with discontinuous topography. J. Comput. Phys. 230 (2011) 5587-5609.

[10] E. Godlewski and P.-A. Raviart, Hyperbolic systems of conservation laws. Ellipses (1991).

[11] S. Gottlieb, C.-W. Shu and E. Tadmor, High order time discretization methods with the strong stability property, SIAM Rev. 43 (2001) 89-112.

[12] S. Karni, Viscous shock profiles and primitive formulations. SIAM J. Numer. Anal. 29 (1992) 1592-1609.

[13] P.G. LeFloch, Entropy weak solutions to nonlinear hyperbolic systems in nonconservative form. Comm. Partial Differential Equations 13 (1988) 669-727. 
[14] T.Y. Hou and P.G. LeFloch, Why nonconservative schemes converge to wrong solutions. Error analysis. Math. Comput. 62 (1994) 497-530.

[15] P.G. LeFloch and M.D. Thanh, The Riemann problem for fluid flows in a nozzle with discontinuous cross-section. Commun. Math. Sci. 1 (2003) 763-797.

[16] P.G. LeFloch, J.M. Mercier and C. Rohde, Fully discrete entropy conservative schemes of arbitrary order. SIAM J. Numer. Anal. 40 (2002) 1968-1992.

[17] R.J. LeVeque, Finite volume methods for hyperbolic problems. Cambridge university press, Cambridge (2002).

[18] T.P. Liu, Shock waves for compressible Navier-Stokes equations are stable. Comm. Pure Appl. Math. 39 (1986) 565-594.

[19] M.L. Munoz Ruiz and C. Pares, Godunov method for non-conservative hyperbolic systems. Math. Model. Num. Anal. 41 (2007) 169-185.

[20] C. Pares and M.J. Castro, On the well-balance property of Roe's method for nonconservative hyperbolic systems. Applications to shallow water equations. Math. Model. Num. Anal. 38 (2004) 821-852.

[21] C. Pares, Numerical methods for non-conservative hyperbolic systems: a theoretical framework. SIAM. J. Num. Anal. 44 (2006) 300-321.

[22] E. Tadmor, The numerical viscosity of entropy stable schemes for systems of conservation laws, I. Math. Comp. 49 (1987) 91-103.

[23] E. Tadmor, Entropy stability theory for difference approximations of nonlinear conservation laws and related time-dependent problems. Acta Numer. 12 (2003) 451-512.

[24] E. Tadmor and W. Zhong, Entropy stable approximations of Navier-Stokes equations with no artificial numerical viscosity. J. Hyperbolic Differ. Equ. 3 (2006) 529-559.

[25] E. Tadmor and W. Zhong, Energy preserving and stable approximations for the two-dimensional shallow water equations, in Mathematics and computation: A contemporary view, Proc. of the third Abel symposium. Ålesund, Norway, Springer (2008) $67-94$.

[26] E. Romenski, D. Drikakis and E. Toro, Conservative models and numerical methods for compressible two-phase flow. J. Sci. Comput. 42 (2010) 68-95. 